\title{
The role of miR-26 in tumors and normal tissues (Review)
}

\author{
JIE GAO and QING-GUANG LIU
}

\author{
Hepatobiliary Surgery Department, the First Affiliated Hospital, Medical School of Xi'an \\ Jiaotong University, Yanta Road 277, Xi'an, Shaanxi 710061, P.R. China
}

Received January 31, 2011; Accepted July 1, 2011

DOI: $10.3892 / \mathrm{ol} .2011 .413$

\begin{abstract}
R-26, a functional miRNA, has received much attention from researchers in recent years. miRNAs may play crucial roles in numerous biological processes such as cell proliferation, apoptosis, tumorigenesis at different stages of non-tumor diseases, growth and development of normal tissues, and other biological processes. The expression of miR-26 has been found to be specific to different biological processes. Furthermore, its expression is frequently abnormal in tumors, indicating that miR-26 may play significant roles in tumor formation. Various reports exist regarding miR-26 involvement in non-tumor diseases, as well as the process of growth and development of normal tissues. In this review, we report findings of recent studies on the expression of miR-26 in different types of diseases and the process of growth and development and its predicted target genes in different tissue types. In conclusion, it is useful for researchers to understand the role of miR-26 in different biological processes.
\end{abstract}

\section{Contents}

1. Processing and general functions of miRNAs

2. Structure and functions of $\mathrm{miR}-26$

3. miRNA-26 and tumors: down- and up-regulation

4. miR-26 and non-tumor disease

5. Relationship between miR-26 and normal tissue growth and development

6. Conclusions

\section{Processing and general functions of miRNAs}

MicroRNAs (miRNAs) are a class of endogenously small (approximately 22-nucleotide) non-coding RNAs present extensively in eukaryotes, which were first discovered in a

Correspondence to: Dr Qing-guang Liu, Hepatobiliary Surgery Department, The First Affiliated Hospital, Medical School of Xi'an Jiaotong University, Yanta Road 277, Xi'an, Shaanxi 710061, P.R. China

E-mail: liuqingguang@vip.sina.com

Key words: miR-26, tumorigenesis growth and development, review
1994 investigation into eelworms. Pri-miRNA (approximately 1000 bp transcription mostly through RNA polymerase II and nearly $20 \%$ of the remaining through RNA polymerase III) forms a distinctive 'hairpin' stem-loop secondary structure and enters the miRNA processing complex. Through a series of processing by Drosha (intranuclear), Exp-5 and Dicer (intracytoplasm), the precursor miRNA (pre-miRNA; approximately 60-70 nt, 5' end phosphorylated $3^{\prime}$ end of the suspended $2 \mathrm{nt}$ sudden) (1) is gradually processed into the miRNA* duplex, and then forms the single-stranded structure of mature miRNA and a miRNA* by helicase. The miRNA* is rapidly degraded. Simultaneously, the mature miRNA is involved in the formation of RNA-induced silencing complex (RISC) sand target mRNA through the 3' untranslated region (UTR) complete complemenary (plants) or imperfect complementary (animals), which mediates target mRNA degradation or translation inhibition, thereby regulating almost $33 \%$ of the protein coding gene (2-5).

The regulation is based on the imperfect complement binding between the seed region of miRNA and 3'-UTR of target mRNA. miRNAs then alter the expression of target genes at transcriptional and post-transcriptional levels, including genes encoding transcription factors and RNA regulatory proteins. The subsequent effects of this process may alter the levels of other mRNA (or protein interaction). miRNA may therefore affect the expression of multiple genes and play a role in a variety of biological processes through transcriptional and post-transcriptional regulation (1). miRNA may change mRNA stability by binding with the 3'-UTR of target mRNA (1). Consequently, researchers have paid substantial attention to miRNA and numerous reports are available on the biogenetic basic function of miRNA and its significant role in disease and normal tissue physiological processes (6-12). Tumor miRNA expression profiles have shown that the tumor has different relatively specific miRNA expression profiles, and that the same miRNA may differ between tumor types (13). miRNAs act as oncogenes or tumor suppressor genes involved in tumorigenesis (14). Calin et al reported that $52.5 \%$ of miRNA genes located in fragile sites are associated with cancer (15). Different miRNA expression profiles exist in different stages of normal tissue differentiation and development processes.

A study regarding single-nucleotide polymorphisms (SNPs) of miRNA in the human genome revealed that miRNA had a lower SNP density compared to the genome average, with only 24 SNPs located in the 325 miRNAs studied. Findings of this study also showed 2 miRNA regions, hsa-mir-26a-2 in the 


\section{miR-26a-1 (22)5' -uucaaguaauccaggauaggcu-3' \\ miR-26a-2 (22)5' -uucaaguaauccaggauaggcu-3' \\ miR-26b (21)5' -uucaaguaauucaggauaggu-3'}

Figure 1. Sequence of mature miR-26.

CTDSP2 gene and hsa-mir-128-1 in the R3HDM1 gene, among a Spanish population (16). Concomitantly, Diederichs and Haber reported that the expression levels of miRNAs were globally reduced in cancer compared with matched normal tissues. A panel of 91 cancer-derived cell lines was analyzed for sequence variations in 15 miRNAs involved in tumorigenesis by virtue of their known target transcripts or their localization to sites of frequent chromosomal instability. One of these miRNAs is $\operatorname{miR}-26$ (17).

miR-26 is a functional miRNA that has merited previous investigation. Various microarray expression profiles showed that miR-26 expression is disordered in a number of human tumors $(18,19)$. The expression of miR-26 is altered during normal tissue growth and development processes such as myogenesis. Consequently, this review summarizes previous investigations into the expression of miR-26 in different types of diseases and different stages of growth and development.

\section{Structure and functions of miR-26}

MiR-26a-1, miR-26a-2 and miR-26b are the only 3 subtypes of the hsa-miR-26 family, and are located in chromosomes 3,12 and 2, respectively. The mature miRNA of miR-26a-1 and miR-26a-2 possesses the same sequence, with the exception of 2 different nucleotides in mature miR-26b (Fig. 1). Pre-miR-26 with stem-loop structure (Fig. 2) processed into mature miR-26 by a series of enzymes of intranuclear and intracytoplasm. The mature miR-26 was $21-22$ nucleotides in length, with a seed region of approximately 6-7 nucleotides. The sequence of miR-26 seed region, an important region for binding to target mRNA, is highly consistent in different genera members. Numerous tumors and normal tissues exhibit different expression of miR-26 during growth, development and tumorigenesis and miR-26 may participate in various biological processes through imperfect sequence complementarity binding between seed region and 3'UTR of target mRNA. miR-26 may repress the target gene translation and decrease expression levels of target gene-coding protein. miRNA has numerous significant target genes in regulatory networks for gene therapy, which renders it important in tumorigenesis and tumor therapy. It has been observed that expression of miR-26 is disordered in many tumors and that it has specific functions in different tumors.

\section{3. miR-26 and tumors: down- and up-regulation}

Increasingly, studies have shown that miRNAs are involved in tumorigenesis and act as oncogenic or tumor-suppressive genes in various tumor types. miRNAs are therefore divided into two
$\begin{array}{llllll}\mathbf{A} & \mathrm{g} & \mathbf{U} & \mathbf{6} & -\mathrm{g} & \mathrm{ca}\end{array}$

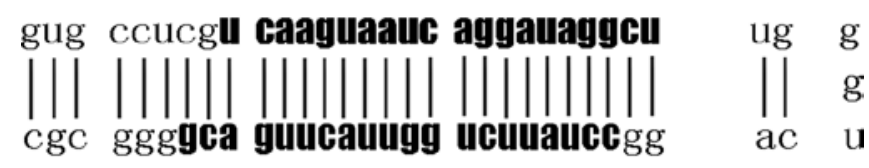

a $\quad$ u $\quad$ gua cc

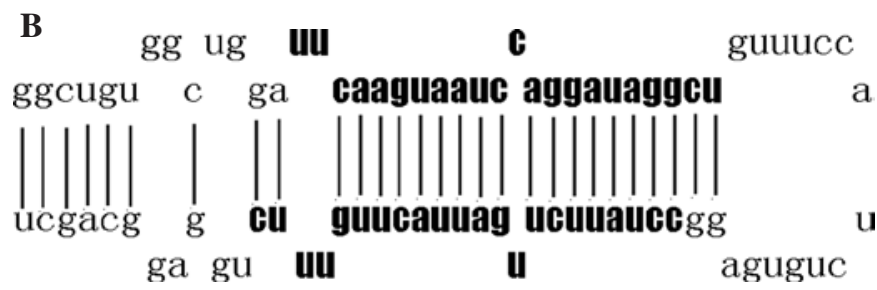

$\mathrm{C}$

ga - u uc u ug

ccgg ccc agu caaguaau aggauagguug $\mathrm{g}$ c

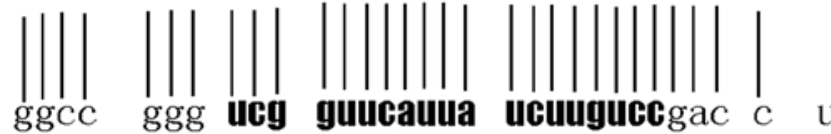

ag $\boldsymbol{B} \ldots$ B6 $\quad \mathrm{ug}^{-}$

Figure 2 Stem-loop structure of pre-miR-26. (A) Pre-miR-26a-1, (B) premiR-26a-2, and (C) pre-miR-26b.

types: oncogenic miRNA (oncomir) such as miR-17-92 cluster, and tumor-suppressive miRNA such as miR-34. As stated above, the expression of miR-26 is disordered in a number of tumors, but its functions remain unknown.

Down-regulation. MiR-26 is down-regulated in various tumor types and may exhibit tumor-suppressive activity during tumorigenesis in these tumors. Wang et al reported that the expression of miR-26 is down-regulated in groups T1 (differentiated grade 1-2) and T2 (differentiated grade 1-3) bladder cancer. miR-26 is the most significant of the 4 down-regulated miRNAs in the T2 group bladder tumors. The decrease in expression of 4 miRNAs is common for all bladder tumors regardless of cancer stage or tumor differentiation. miR-26 may therefore be a significant marker in bladder cancer (20). Maillot et al observed that E2-repressed miR-26a and miR181a regulated numerous genes associated with cell growth and proliferation through estrogen receptors and transcription factors. Additionally, miRNA expression was regulated in breast cancer in women who had received anti-estrogen neoadjuvant therapy. This regulation indicated that miRNA was correlated to anti-estrogen resistance of breast cancer (21). Zhang et al noted that miR-26a was down-regulated in breast cancer specimens and cell lines, and that it initiated apoptosis through endogenous and exogenous pathways activated by caspase-8 and 9 as well as through binding to the 3'-UTR of MTDH and EZH2 directly. MiR-26 impairs the in vitro colonyforming and in vivo tumor-loading abilities of MCF7 cells (22). Moreover, Yu et al found that the expression of miR-26 in oral squamous cell carcinoma in Syrian hamsters was decreased (23). Visone et al reported that miR-26a was significantly decreased in anaplastic carcinomas (ATC) in comparison 
to normal thyroid tissue. The overexpression of miR-26 in 2 human ATC-derived cell lines significantly decreased thyroid carcinogenesis, suggesting a crucial role for miR-26a down-regulation in thyroid carcinogenesis. miR-26a may exhibit a tumor-suppressive activity since cell-growth inhibition was achieved (24). In their study, Ciarapica et al compared a group of 5 rhabdomyosarcoma (RMS1-5) with two muscle tissues as controls (MT1 and MT2). These authors observed that miR-26a exhibited a significant negative fold-change in all RMS1-5 compared with MT1 and MT2, indicating that its down-regulation has a potential role in rhabdomyosarcoma. They also confirmed that EZH2, a validated target gene of miR-26a, was up-regulated in rhabdomyosarcoma, indicating that a miR-26a-dependent regulation of EZH2 may be active in rhabdomyosarcoma cells (25).

Myc is a significant oncogene that is always mutated or amplified in various types of human cancer. Myc is associated with cell growth and proliferation and is crucial to tumorigenesis and progression. Following the study of cell lines and murine lymphoma models (26), a study on miRNA expression profiles in Myc-driven tumorigenesis reported that the expression of miR-26 decreased in Burkitt lymphoma (BL; an aggressive variant of non-Hodgkin's B-cell lymphoma). Overexpression of miR-26a in human BL-derived cell lines using the episomal expression system produces an increased percentage of cells in the G1 phase and fewer cells in the S/G2 phase within the miR-26a-expressing cell lines as compared to the empty vector controls $72 \mathrm{~h}$ after transfection, indicating that miR-26 arrested cell cycle progression. miR-26a was consistently repressed by Myc in multiple tumors, indicating that this miRNA may have a strong tumor-suppressor function in Myc-induced lymphomas. These authors attempted to elucidate the effector pathway for miR-26a. The focus was on potential targets, nominated in at least 2 different databases, which may have been involved in the observed G1 arrest by miR-26a overexpression. The results showed that a significant degression of EZH2 (Enhancer of Zeste Homolog 2) in the gene expression profile was induced by miR-26a over-expression, in both human BL-derived and murine lymphoma cell lines. Myc may thus contribute to the up-regulation of EZH2 via the down-regulation of its targeting miRNA. The suppression of the miR-26a-mediated attenuation of EZH2 expression by Myc was shown to play a critical role in lymphomagenesis. A positive feedback loop comprising Myc and EZH2 was involved in the formation of the malignant lymphoma phenotype (26). Kota et al reported that the expression of miR-26 was down-regulated in hepatocellular carcinoma (HCC) cells and that overexpression of miR-26a in liver cancer cells in vitro induced an increase in cells of G1 stage as well as a decrease in cells of the S stage, indicating that miR-26a induced a G1 arrest. Systemic administration of this miRNA to a mouse model of HCC using adeno-associated virus resulted in the inhibition of cancer cell growth and proliferation, and increased tumor-specific apoptosis. This process indicated that miR-26a was a tumor-suppressor miRNA (27). A study is available pertaining to miRNA expression, survival and response to interferon in 455 patients with HCC who had undergone radical tumor resection. Expression of miR-26a and miR-26b was found to be higher in female than in male individuals in the non-tumor liver tissue of hepatocellular carcinoma patients, indicating that a higher expression of
miR-26 may explain the lower morbidity. Expression of miR-26 was down-regulated in tumors compared with paired noncancerous tissues, indicating that the sexpression of miR-26 was associated with HCC. Moreover, tumors with a reduced miR-26 expression exhibited a distinct transcriptomic pattern and activated the signaling pathways between nuclear factor $\kappa \mathrm{B}$ and interleukin-6, which may play a role in tumor development according to gene networks information. Patients with a lower miR-26 expression in HCC had a shorter survival but a more favorable response to interferon therapy than those with a higher miR-26 expression in HCC, indicating that miR-26 was associated with post-operative survival (28).

The studies of miR-26 expression profiles in tumors raise 3 significant points. First, miR-26 expression decreases in bladder tumor, breast cancer, oral squamous cell carcinoma, anaplastic carcinomas, Burkitt lymphoma HCC and rhabdomyosarcoma, and it may be a suppressor miRNA in those tumors. Second, Myc is a significant oncogene associated with tumorigenesis and is always mutated or amplified in various types of human cancer. miR-26a is one of the miRNAs consistently repressed by Myc in multiple tumors (26). Third, the 3'-UTR of EZH2, a crucial subunit of Polycomb repressive complex 2, has binding sites with a seed region of miR-26. EZH2 is an important transcription regulation factor in tumorigenesis, and since it regulates the global level of gene expression it may be one of the downstream target genes of miR-26 $(26,27)$. The expression of EZH2 is up-regulated in numerous tumor types and miR-26 may exhibit functions of tumor suppression via downregulation of the translation of $\mathrm{EZH} 2$.

Up-regulation. miR-26 expression reportedly decreased in various tumor types, where it functioned as tumor-suppressor miRNA. However, various recent studies revealed that the expression of miR-26 was up-regulated in tumors such as glioma $(29,30)$. Huse et al reported that miR-26a was overexpressed in high-grade glioma (the most prevalent diagnostic category of primary brain tumor in the adult population) and directly targeted PTEN. MiR-26a was frequently amplified at the DNA level in a subset of human high-grade gliomas and its over-expression was strongly associated with monoallelic PTEN loss. Overexpression of miR-26a in a murine glioma model using the RCAS/tv-a system revealed that miR-26a repressed the endogenous PTEN protein effectively by binding to 3 potential binding sites in the PTEN 3'-UTR in a relevant glioma model system, promoting tumorigenesis. miR-26 may therefore be an oncomir in glioma. Notably, the study by Huse et al indicated that miR-26a over-expression in LN-18 cells also decreased the expression of EZH2 and SMAD1, indicating that the transcripts of the two proteins were effectively targeted by miRNA during gliomagenesis (29). This review suggests that miR-26 regulates numerous target genes simultaneously and that its role is completely different in certain tumors. In their study on an oncomir/oncogene cluster regulating glioblastoma survivorship, Kim et al noted that miR-26a regulated PTEN expression and AKT activation and inhibited RB1 and MAP3K2/MEKK2 expression and JNK-dependent apoptosis in glioblastoma multiforme (GBM). PTEN was therefore considered to be one of the downstream target genes of miR-26a in GBM. Overexpression of miR-26a increased GBM cell growth compared to the control cells, consistent with the characteristics of AKT activation and miR-26 
overexpression by the miR-26 mimic or lentiviral in U87 GBM cells, lack of functional PTEN, decreased RB1 expression and increased DNA synthesis. The MAP3K2 gene encodes MEKK2, which is involved in JNK and ERK5 activation, and JNK activation can promote apoptosis in GBM cells. miR-26a therefore decreased JNK-dependent apoptosis by inhibiting MAP3K2/MEKK2 expression in GBM cells, whereas the miR-26a inhibitor increased this expression. PTEN was therefore not the only target gene of miR-26a in GBM (30).

The above-mentioned studies regarding miR-26 expression in glioma raise 3 significant points. First, miR-26a expression increases in GBM and promotes tumor cell growth and proliferation function as an oncomir in GBM. Second, PTEN may be one of the downstream target genes of miR-26a in GBM, due to the 3 potential binding sites with miR-26a in its 3'-UTR. miR-26a regulates PTEN expression and AKT activation and inhibits RB1 and MAP3K2/MEKK2 expression and JNK-dependent apoptosis in GBM. Third, miR-26 regulates the expression of a set of target genes and the role of these genes may be completely different in specific tumors. The involvement of miR-26 in these tumors therefore depends on those target genes and their corresponding pathways.

\section{4. miR-26 and non-tumor disease}

miR-26 expression is not only disordered in tumorigenesis but also alterable in non-tumor diseases. Primary billiary cirrhosis (PBC) caused by chronic cholestasis is often accompanied by autoimmune diseases such as rheumatoid arthritis and scleroderma. Padgett et al observed that a total of 35 independent miRNAs in the miRNA expression profile are disordered and that miR-26a is one of the down-regulated miRNAs. The predicted targets of these alternative miRNAs are known to affect cell proliferation, apoptosis, inflammation, oxidative stress and metabolism associated with the development of PBC (31). The roles that miR-26 plays in non-tumor diseases have yet to be clarified, and further studies are required.

\section{Relationship between miR-26 and normal tissue growth and development}

miRNAs play crucial roles in numerous biological processes via their target genes. It is known that miR-26 plays a significant role in the growth, development and cell differentiation of different tissues. Murine fetal hepatoblast cells can be induced to differentiate between the hepatocyte and cholangiocyte, and the expression of miRNAs is altered during the differentiation processes. MiR-23b cluster miRNAs including miR-26a have a gradient of effects on cell fate choice in the fetal mouse liver via transforming growth factor- $\beta$ (TGF $\beta$ )/ bone morphogenetic protein signal pathway. Low levels of the miR-23b miRNAs are required in cholangiocytes to allow TGF $\beta$ signaling and bile duct formation (32). In osteogenesis several miRNAs (for example miR-26a) regulate osteoblast cell growth and differentiation in human adipose tissue-derived stem cells (33). The function of miR-26 in myogenesis is clearest in studies of miRNA in normal tissue growth and development. Wong and Tellam (34) investigated the miRNA expression profile of myogenesis. These authors identified 6 miRNAs with 2-fold or greater significant expres-
Table I. Expression of miR-26 in tumors.

\begin{tabular}{lll}
\hline & \multicolumn{1}{c}{ Down-regulated } & Up-regulated \\
\hline Tumor & $\begin{array}{l}\text { Bladder tumor } \\
\text { Breast cancer } \\
\text { Oral squamous cell carcinoma }\end{array}$ & Glioma \\
& $\begin{array}{l}\text { Anaplastic carcinomas } \\
\text { Burkitt lymphoma }\end{array}$ & \\
& Hepatocellular carcinoma & \\
& Rhabdomyosarcoma & \\
Function & $\begin{array}{l}\text { Tumor suppressive } \\
\text { function }\end{array}$ & Oncogenic \\
Target gene & EZH2 & activity \\
\hline
\end{tabular}

sion alteration in myotubes. These miRNAs were divided into 3 groups according to the level of expression. MiR-26a, an up-regulated miRNA in Cluster II, was up-regulated more gradually during the course of myogenesis. Overexpression of miR-26a in murine myogenic $\mathrm{C} 2 \mathrm{C} 12$ cells induced creatine kinase activity, an enzyme that markedly increased during myogenesis. myoD and myogenin mRNA expression levels were also up-regulated, and EZH2 was identified as a potential target of miR-26a. Overexpression of miR-26a decreased EZH2 mRNA expression and suppressed the activity of a luciferase reporter construct fused with the 3'UTR of EZH2. The up-regulated expression of miR-26a was required during terminal differentiation, thus the negative regulator of myogenesis EZH2 is rapidly and efficiently silenced, thereby promoting myogenesis and terminal differentiation (34). miR-26 was found to play a role in normal tissue growth and development and to have an impact upon cell proliferation and differentiation; however, the mechanism remains to be clarified.

\section{Conclusions}

miR-26, a functional miRNA, has merited investigation, and has been found to possess different roles in different tumors. The development of microarray techniques has led to significant progress in the investigation of the expression of miR-26 in numerous tumor types and certain normal tissue growth and development. However, the target genes and molecule mechanisms remain to be elucidated. The expression of miR-26 has been found to decrease in bladder and breast cancer, oral squamous cell carcinoma, anaplastic carcinomas, Burkitt lymphoma, HCC and rhabdomyosarcoma, and is considered to be a suppressor miRNA in those tumors. Expression of miR-26 increased in GBM, where it promoted tumor cell growth and proliferation as an oncomir. MiR-26 is instrumental to normal tissue growth and development by impacting on cell proliferation and differentiation. Various studies also showed that EZH2, PTEN, SMAD1 and MTDH are potential downstream target genes of miR-26. In conclusion, the molecular mechanisms of miR-26 and the target genes in different tumors remain unclear and should be investigated. 


\section{References}

1. Liu X, Chen Z, Yu J and Zhou X: MicroRNA profiling and head and neck cancer. Comp Funct Genomics 837514: 1-11, 2009.

2. Lewis BP, Shih I, Jones-Rhoades MW, Bartel DP and Burge CB: Prediction of mammalian microRNA targets. Cell 115: 787-798, 2003.

3. Lewis BP, Burge CB and Bartel DP: Conserved seed pairing, often flanked by adenosines, indicates that thousands of human genes are microRNA targets. Cell 120: 15-20, 2005.

4. Xiaohui X, Jun L, Kulbokas EJ, et al: Systematic discovery of regulatory motifs in human promoters and 3' UTRs by comparison of several mammals. Nature 434: 338-345, 2005.

5. Williams AE: Functional aspects of animal microRNAs. Cell Mol Life Sci 65: 545-562, 2008.

6. Stefani G and Slack FJ: Small non-coding RNAs in animal development. Nat Rev Mol Cell Biol 9: 219-230, 2008.

7. Filipowicz W, Bhattacharyya SN and Sonenberg N: Mechanisms of post-transcriptional regulation by microRNAs: are the answers in sight? Nat Rev Genet 9: 102-114, 2008.

8. Bartel DP: MicroRNAs: genomics, biogenesis, mechanism and function. Cell 116: 281-297, 2004.

9. Bushati N and Cohen SM: microRNA functions. Annu Rev Cell Dev Biol 23: 175-205, 2007.

10. Chang TC and T Mendell J: microRNAs in vertebrate physiology and human disease. Annu Rev Genomics Hum Genet 8: 215-239, 2007.

11. Kloosterman WP and Plasterk RHA: The diverse functions of microRNAs in animal development and disease. Dev Cell 11: 441-450, 2006

12. Ambros V: The functions of animal microRNAs. Nature 431 350-355, 2004.

13. Tong AW and Nemunaitis J: Modulation of miRNA activity in human cancer: a new paradigm for cancer gene therapy? Cancer Gene Ther 15: 341-355, 2008.

14. Chen CZ: MicroRNAs as oncogenes and tumor suppressors. N Engl J Med 353: 1768-1771, 2005

15. Calin GA, Sevignani C, Dumitru CD, et al: Human microRNA genes are frequently located at fragile sites and genomic regions involved in cancers. Proc Natl Acad of Sci U S A 101: 2999-3004, 2004.

16. Muinos-Gimeno M, Montfort M, Bayes M, Estivill X and Espinosa-Parrilla Y: Design and evaluation of single-nucleotide polymorphisms in microRNA genomic regions for association studies in human disease. Eur J Hum Genet 18: 218-226, 2010.

17. Diederichs $S$ and Haber DA: Sequence variations of microRNAs in human cancer: alterations in predicted secondary structure do not affect processing. Cancer Res 66: 6097-6104, 2006.

18. Calin GA and Croce CM: MicroRNA signatures in human cancers. Nat Rev Cancer 6: 857-866, 2006.

19. Marilena V Iorio, Ferracin M, Liu CG, et al: MicroRNA gene expression deregulation in human breast cancer. Cancer Res 65: 7065-7070, 2005.
20. Wang G, Zhang $\mathrm{H}$, He $\mathrm{H}$, et al: Up-regulation of microRNA in bladder tumor tissue is not common. Int Urol Nephrol 42: 95-102, 2009

21. Maillot G, Lacroix-Triki M, Pierredon S, et al: Widespread estrogen-dependent repression of microRNAs in breast tumor cell growth. Cancer Res 69: 8332-8340, 2009.

22. Zhang B, Liu XX, He JR, et al: Pathologically decreased miR-26a antagonizes apoptosis and facilitates carcinogenesis by targeting MTDH and EZH2 in breast cancer. Carcinogenesis 32: 2-9, 2010

23. Yu T, Wang XY, Gong RG, et al: The expression profile of microRNAs in a model of 7,12-dimethyl-benz[a]anthranceinduced oral carcinogenesis in Syrian hamster. J Exp Clin Cancer Res 28: 64-73, 2009.

24. Visone R, Pallante P, Vecchione A, et al: Specific microRNA are downregulated in human thyroid anaplastic carcinomas. Oncogene 26: 7590-7595, 2007.

25. Ciarapica R, Russo G, Verginelli F, Donfrancesco A, Rota R and Giordano A: Deregulated expression of miR-26a and EZH2 in rhabdomyosarcoma. Cell Cycle 8: 172-175, 2009.

26. Sandrine S, Lars B and Thomas W: Repressing the repressor: a new mode of MYC action in lymphomagenesis. Cell Cycle 8: 556-559, 2009.

27. Kota J, Chivukula RR, O'Donnell KA, et al: Therapeutic microRNA delivery suppresses tumorigenesis in a murine liver cancer model. Cell 137: 1005-1017, 2009.

28. Ji J, Shi J, Budhu A, et al: MicroRNA expression, survival, and response to interferon in liver cancer. N Engl J Med 361: 1437-1447, 2009.

29. Huse JT, Brennan C, Hambardzumyan D, et al: The PTEN-regulating microRNA miR-26a is amplified in highgrade glioma and facilitates gliomagenesis in vivo. Genes and Dev 23: 1327-1337, 2009

30. Kim H, Huang W, Jiang X, Pennicooke B, Park PJ and Johnson MD: Integrative genome analysis reveals an oncomir/ oncogene cluster regulating glioblastoma survivorship. Proc Natl Acad of Sci USA 107: 2183-2188, 2010.

31. Padgett KA, Lan RY, Leung PC, et al: Primary billiary cirrhosis is associated with altered hepatic microRNA expression. J Autoimmun 32: 246-253, 2009

32. Rogler CE, LeVoci L, Ader T, Massimi A, Tchaikovskaya T, Norel $\mathrm{R}$ and Rogler LE: MicroRNA-23b cluster microRNA regulate transforming growth factor-beta/bone morphogenetic protein signaling and liver stem cell differentiation by targeting Smads. Hepatology 50: 575-584, 2009.

33. Itoh T, Nozawa Y and Akao Y: MicroRNA-141 and -200a are involved in bone morphogenetic protein-2-induced mouse preosteoblast differentiation by targeting distal-less homeobox 5 . J Biol Chem 284: 19272-19279, 2009.

34. Wong CF and Tellam RL: MicroRNA-26a targets the histone methyltransferase enhancer of Zeste homolog 2 during myogenesis. J Biol Chem 283: 9836-9843, 2008. 